\title{
Supporting Quality of Service in Energy Harvesting Wireless Links: The Effective Capacity Analysis
}

\author{
Jie Gong, Shan Zhang, Xiaolei Wang, Sheng Zhou, Zhisheng Niu \\ Tsinghua National Laboratory for Information Science and Technology \\ Department of Electronic Engineering \\ Tsinghua University, Beijing 100084, China \\ gongj13@mail.tsinghua.edu.cn, \{zs89125, vicharewang\}@gmail.com, \{sheng.zhou, niuzhs $\} @$ tsinghua.edu.cn
}

\begin{abstract}
This paper studies the quality of service (QoS) performance of wireless links powered by energy harvesting techniques with finite energy battery. We adopt a discretized energy arrival and power allocation model and a finite state Markov chain (FSMC) channel model, and hence, the service process can be modeled as a FSMC. The effective capacity for given power allocation policies is analyzed to characterize the QoS performance of energy harvesting wireless links. Based on the analysis, we evaluate the effective capacity of some commonly used power allocation policies compared with nonenergy harvesting system via numerical simulations. It is shown that the performance degrades due to the randomness of energy arrival, and the constant-greedy policy achieves the highest effective capacity among all the considered policies in the high SNR regime.
\end{abstract}

\section{INTRODUCTION}

The future wireless communication networks are targeted at minimizing the $\mathrm{CO}_{2}$ emissions for green communications while at the same time satisfying diverse quality of service (QoS) requirements such as high data rate, short delay, and small delay-violation probability. Exploring the renewable energy (e.g. solar or wind energy) to power the wireless devices, namely the energy harvesting technology, is a promising solution to achieve environmental-friendly green communications. However, due to the randomness of the energy arrival process as well as the battery capacity limitations, providing QoS guarantee for users by energy harvesting technology is a challenging issue. Specifically, besides the interaction between the channel dynamics and the data queue variation, the dynamics of energy buffer introduces another dimension (energy domain) of difficulties for the QoS performance analysis.

In recent years, there have been efforts studying the energy harvesting wireless systems. Ref. [1] has proposed a throughput optimal power allocation policy and an average delay optimal policy in additive white Gaussian noise (AWGN) channel. Later, the capacity of energy harvesting links with infinite battery size is determined in [2] via information theory. Very recently, the capacity with finite battery size is studied in [3]. In the fading channel, directional water-filling (WF) policy

This work is sponsored in part by the National Science Foundation of China (NSFC) under grant No. 61201191, the National Basic Research Program of China (973 Program: 2012CB316001), the Creative Research Groups of NSFC under grant No. 61321061, and Hitachi R\&D Headquarter.
[4] is proved throughput optimal for greedy source, and the solution is extended to multiple antenna transceiver case [5], where a spatial-temporal WF policy is proposed. Further in [6], the authors consider data arrival with hybrid power supply, and propose an optimal reverse multi-stage WF policy. But these policies assume that all the energy arrival, the channel fading and the data arrival is explicitly known, which requires strictly accurate predictions. In addition, most of the existing work mainly focuses on the throughput maximization and/or capacity achieving problems, while how to guarantee users' QoS such as delay remains unsolved.

To address this problem, we need to characterize the energy harvesting wireless links in terms of QoS metrics. The effective capacity model proposed in [7] is an efficient tool to analyze the statistical QoS performance such as delay-bound violation probability of various channels. For uncorrelated channel, there is a simplified expression of effective capacity, and optimal power and rate adaptation for QoS guarantees can be obtained [8]. Also, power control and power-delay tradeoffs are studied in [9] and [10], respectively. For correlated channels, ref. [11] studies the effective capacity of the finite state Markov chain (FSMC) channel, and ref. [12] analyzes that of the correlated rayleigh fading channel. By properly interpreting the energy arrival and departure dynamics similar to the channel dynamics, it can also be used to analyze the QoS performance of energy harvesting systems.

In this paper, we study the QoS guarantee performance of the energy harvesting wireless links. Specifically, we consider a single wireless link with renewable energy powered transmitter. We model the energy arrival, storage and usage as a discrete model, where one unit of energy is regarded as a "packet", and model the channel by FSMC. We derive the effective capacity formula for the power allocation policies which can guarantee that the system states are irreducible. Based on the formula, we evaluate the performance of several commonly used policies, including greedy policy, constant power policy, throughput optimal policy and so on. The influence of energy harvesting on QoS performance compared with constant power supply is also studied.

The rest of the paper is organized as follows. Section II introduces the concept of effective capacity. System model is presented in Section III. Based on this, Section IV analyzes the 
effective capacity of given power allocation policies, which are then evaluated in Section V via numerical simulations. Finally, Section VI concludes the paper.

\section{EFFECTIVE CAPACITY AND Statistical QoS GUARANTEE}

Effective capacity is defined as the maximal constant arrival rate that a given service process can support to guarantee a certain QoS requirement [7]. Specifically, given a stationary and ergodic stochastic service process $\left\{R_{t}, t=1,2, \ldots\right\}$, define $S_{t}=\sum_{i=1}^{t} R_{i}$ as the partial sum of $R_{t}$. Then the effective capacity, denoted by $\alpha_{E C}(\theta)$, is given by [7, Eq. (12)]

$$
\alpha_{E C}(\theta)=-\lim _{t \rightarrow \infty} \frac{1}{\theta t} \log \left(\mathbb{E}\left(e^{-\theta S_{t}}\right)\right),
$$

where $\theta$ is a parameter reflecting the QoS constrains, and $\mathbb{E}$ is the expectation operator. Based on large deviation theory, for a queuing system with infinite buffer, when the constant arrival rate is no larger than $\alpha_{E C}(\theta)$, its queue-length process $Q_{t}$ converges in distribution to a random variable $Q_{\infty}$ so that:

$$
-\lim _{x \rightarrow \infty} \frac{\log \left(\operatorname{Pr}\left\{Q_{\infty}>x\right\}\right)}{x}=\theta
$$

which means the probability of the queue-length exceeding $x$ decays exponentially with $x$ at speed $\theta$. Generally, larger $\theta$ corresponds to a faster decay rate (i.e., a more stringent QoS requirement) and a smaller effective capacity (i.e., a lower supportable arrival rate). Thus, parameter $\theta(\theta>0)$ is called the $Q o S$ exponent [7]. Specially, when $\theta \rightarrow \infty$, no delay is allowed and $\alpha_{E C}(\theta)$ equals to the minimal service rate (real-time service); when $\theta \rightarrow 0$, any delay is acceptable and $\alpha_{E C}(\theta)$ equals to the average service rate (best effort service).

Therefore, the theory of effective capacity has offered a method to quantify the relationship between the delay constraint and the service capability of an time varying service process. Based on this theory, in this paper, we analyze the QoS guarantee performance of energy harvesting systems in terms of effective capacity.

\section{SySTEM MODEL}

We consider a single-link wireless communication system where the transmitter is powered by renewable energy. The renewable energy is harvested from ambient energy sources and stored in a energy battery with a finite capacity denoted by $E_{\text {max }}$. Assume the system is slotted with frame length $T_{f}$. The state transition depicted in Fig. 1. In frame $t$, the system state is denoted as $S_{t}=\left(E_{t}, C_{t}\right)$, where $E_{t}$ is the amount of energy is the battery, and $C_{t}$ is the channel state. We assume the channel state is known by the transmitter through error-free channel estimation and feedback. The state transition happens between two consecutive frames. We divide the state transition process into three steps for the ease of description. At the beginning of the frame $t$, the power allocation policy determines the amount of energy $U_{t}\left(\leq E_{t}\right)$ used during the frame $t$ depending on the system state $S_{t}$. Hence, the transitional energy battery state denoted by $\bar{E}_{t}$ is updated as $\bar{E}_{t}=E_{t}-U_{t}$. By the end of frame $t$, the energy battery state is updated by adding the

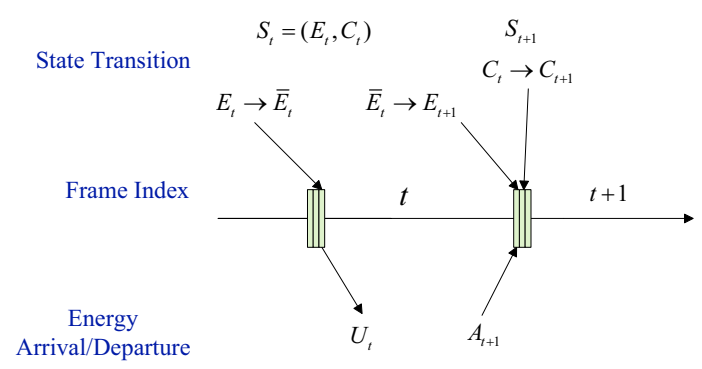

Fig. 1. State transition of a single-link energy harvesting system.

amount of arrived energy $A_{t+1}$, and we denote the energy battery state as $E_{t+1}=\min \left\{\bar{E}_{t}+A_{t+1}, E_{\max }\right\}$. Finally, the channel state transits from $C_{t}$ to $C_{t+1}$. As a result, the overall energy battery state transition can be expressed as

$$
E_{t+1}=\min \left\{E_{t}-U_{t}+A_{t+1}, E_{\max }\right\} .
$$

We consider a discrete system model, where the energy is discretized with unit $\Delta E$. The energy battery capacity can be denoted by $E_{\max }=N$, indicating that the maximum energy quantity $N \Delta E$ can be stored. Then, the battery energy state can be denoted by $E_{t}=n, n \leq N$, and the energy usage is constrained by $U_{t} \in\left\{0,1, \ldots, E_{t}\right\}$.

\section{A. Energy arrival model}

The harvested energy is assumed a stationary and ergodic Markov process. In accordance with the discrete energy battery model, the energy arrival is denoted by $A_{t} \in\{0,1, \ldots\}$. The energy battery state transition between $\bar{E}_{t}$ and $E_{t+1}$ can be denoted by an $(N+1) \times(N+1)$ matrix

$$
\mathbf{P}_{A}=\left(\begin{array}{cccc}
P_{A}(0,0) & P_{A}(0,1) & \ldots & \bar{P}_{A}(0, N) \\
0 & P_{A}(1,1) & \ldots & \bar{P}_{A}(1, N) \\
\vdots & \vdots & \ddots & \vdots \\
0 & 0 & \ldots & \bar{P}_{A}(N, N)
\end{array}\right)
$$

where $P_{A}(i, j)$ denotes the transition probability from state $\bar{E}_{t}=i$ to $E_{t+1}=j$ by harvesting energy $(j-i) \Delta E$. As the harvested energy is non-negative, the matrix $\mathbf{P}_{A}$ is an upper triangular matrix where $P_{A}(i, j)=0, \forall j<i$. As we assume a finite battery size, and the energy arrival may exceed the battery capacity. So the last column of $\mathbf{P}_{A}$ is obtained as

$$
\bar{P}_{A}(i, N)=1-\sum_{j=0}^{N-1} P_{A}(i, j) . \quad i=0,1, \ldots, N
$$

In this paper, we adopt the Poisson process with parameter $\lambda$ to model the energy arrival. In this case, the state transition probability due to energy arrival is calculated as

$$
P_{A}(i, j)=\frac{\left(\lambda T_{f}\right)^{j-i} \exp \left(-\lambda T_{f}\right)}{(j-i) !}, \quad i \leq j<N
$$

\section{B. Channel model}

The channel is assumed to be block fading, i.e., the channel state is static during a transmitting frame, while variable among frames. The received signal-to-noise ratio (SNR) in each frame under a reference static transmit power $P_{0}$ is 
assumed to follow Nakagami- $m$ distribution [13], which is a generalized model for mobile multipath propagation. By choosing different values of $m$, it can represent different fading channels. For instance, the Rayleigh fading channel model is obtained if $m=1$. The probability density function of received SNR in the Nakagami- $m$ model is,

$$
p_{\Gamma}(\gamma)=\frac{m^{m} \gamma^{m-1}}{\bar{\gamma}^{m} \Gamma(m)} \exp \left(-\frac{m \gamma}{\bar{\gamma}}\right),
$$

where $\bar{\gamma}$ is the average received SNR with transmit power $P_{0}$, and $\Gamma(m)=\int_{0}^{+\infty} t^{m-1} \exp (-t) \mathrm{d} t$ is the Gamma function.

To model the discrete wireless channel, the FSMC model [14] is adopted. The entire SNR range is divided into $M$ nonoverlapping consecutive segments with $M+1$ boundary points $\gamma_{1}<\gamma_{2}<\ldots<\gamma_{M+1}$, where $\gamma_{1}=0$ and $\gamma_{M+1}=+\infty$. Consequently, there are a total of $M$ channel states, denoted by $C_{t} \in\{1,2, \ldots, M\} . C_{t}=i, i=1,2, \ldots, M$ means that the SNR $\gamma \in\left[\gamma_{i}, \gamma_{i+1}\right)$. Each of the channel states can only transit to its adjacent state, i.e., $P_{C}(i, j)=0,|i-j| \geq 2$. Hence, the state transition probability matrix can be expressed as

$$
\mathbf{P}_{C}=\left(\begin{array}{cccc}
P_{C}(1,1) & P_{C}(1,2) & \ldots & 0 \\
P_{C}(2,1) & P_{C}(2,2) & P_{C}(2,3) & \\
\vdots & \vdots & \ddots & \vdots \\
0 & \ldots & P_{C}(M, M-1) & P_{C}(M, M)
\end{array}\right) .
$$

The elements of (8) can be calculated as follows [15]:

$$
\begin{aligned}
& P_{C}(i, i+1)=\frac{N_{i+1} T_{f}}{\pi_{i}}, \quad i=1,2, \ldots, M-1 \\
& P_{C}(i, i-1)=\frac{N_{i} T_{f}}{\pi_{i}}, \quad i=2,3, \ldots, M
\end{aligned}
$$

where

$$
\pi_{i}=\int_{\gamma_{i}}^{\gamma_{i+1}} p_{\Gamma}(\gamma) \mathrm{d} \gamma
$$

is the probability that the received SNR lies in $\left[\gamma_{i}, \gamma_{i+1}\right)$, and

$$
N_{i}=\sqrt{2 \pi \frac{m \gamma_{i}}{\bar{\gamma}}} \frac{f_{d}}{\Gamma(m)}\left(\frac{m \gamma_{i}}{\bar{\gamma}}\right)^{m-1} \exp \left(-\frac{m \gamma_{i}}{\bar{\gamma}}\right)
$$

is the cross rate of mode $m$ [16], where $f_{d}$ denotes the Doppler spread of the channel. The diagonal elements can then be calculated as $P_{C}(i, i)=1-\sum_{j \neq i} P_{C}(i, j)$.

\section{EfFective CAPACITy of Policies in Energy HARVESTING SYSTEMS}

In this section, we describe the power allocation policies in state transition form to obtain the overall state transition matrix. Then we analyze the effective capacity of energy harvesting systems under these policies.

\section{A. Power allocation policies}

We consider the power allocation policies that depend only on the current system state, and express them in matrix form. Note that the power allocation only changes the energy battery state from $E_{t}$ to $\bar{E}_{t}$. As a result, the transition between the state $\left(E_{t}, C_{t}\right)$ and $\left(\bar{E}_{t}, C_{t}\right)$ is

$$
\mathbf{P}_{U}=\left(\begin{array}{cccc}
\tilde{\mathbf{P}}_{U}(0,0) & 0 & \ldots & 0 \\
\tilde{\mathbf{P}}_{U}(1,0) & \tilde{\mathbf{P}}_{U}(1,1) & \ldots & 0 \\
\vdots & \vdots & \ddots & \vdots \\
\tilde{\mathbf{P}}_{U}(N, 0) & \tilde{\mathbf{P}}_{U}(N, 1) & \ldots & \tilde{\mathbf{P}}_{U}(N, N)
\end{array}\right)
$$

where $\tilde{\mathbf{P}}_{U}(i, j)=\operatorname{diag}\left\{P_{U}(i, j, 1), \ldots, P_{U}(i, j, M)\right\}$ is a diagonal matrix where $P_{U}(i, j, k)$ is the transition probability from $(i, k)$ to $(j, k)$ by utilizing $U_{t}=i-j$ units of energy. Hence, the matrix $\mathbf{P}_{U}$ is a block lower triangular matrix where each block is an $M \times M$ diagonal matrix. The elements satisfy

$$
\begin{aligned}
& P_{U}(i, j, k)=0, \forall j>i, \\
& \sum_{j=0}^{N} P_{U}(i, j, k)=1 .
\end{aligned}
$$

We consider deterministic power allocation policies, i.e.,

$$
P_{U}(i, j, k) \in\{0,1\},
$$

which together with (15) decides that there is and only is one element equaling 1 in each row. With this model, general randomized policies where $P_{U}(i, j, k) \in[0,1]$ can be analyzed, which is left for future work.

Note that we assume the system transmits dummy packets when the data queue is empty. As a result, we lost some service capability as the dummy packet transmission wastes energy. Hence, the policies modeled in this paper can only achieve a lower-bound service capacity.

\section{B. Overall state transition and data rate}

Given the power allocation policy matrix, the transition probability matrix of the overall system state $S_{t}$ is

$$
\mathbf{P}_{S}=\mathbf{P}_{U}\left(\mathbf{P}_{A} \otimes \mathbf{P}_{C}\right)
$$

where $\otimes$ is the Kronecker product [17, Sec. 4.2.1]. As a consequence, $\mathbf{P}_{S}$ is an $(N+1) M \times(N+1) M$ matrix with elements $P_{S}(M i+p, M j+q)=\sum_{k=0}^{\min \{i, j\}} P_{U}(i, k, p) P_{A}(k, j) P_{C}(p, q)$, where $i, j \in\{0,1, \ldots, N\}$ is the energy battery state, and $p, q \in\{1,2, \ldots, M\}$ is the channel state.

We assume the harvested energy is used as transmit power only, i.e., the processing power consumption is ignored. In this case, the transmit power is obtained by $P_{T}\left(E_{t}, C_{t}\right)=$ $U_{t} \Delta E / T_{f}$. For a given power allocation policy $\mathbf{P}_{U}$, we calculate the achievable rate in state $\left(E_{t}, C_{t}\right)=(i, j)$ according to adaptive MQAM [18] by averaging the rate over the SNR range $\left[\gamma_{j}, \gamma_{j+1}\right)$ :

$$
\begin{aligned}
\left.R(M i+j)\right|_{\mathbf{P}_{U}}= & \frac{T_{f} W}{\pi_{j}} \int_{\gamma_{j}}^{\gamma_{j+1}} \log _{2}(1+ \\
& \left.\frac{1.5}{-\log \left(5 P_{e}\right)} \frac{P_{T}(i, j) \gamma}{P_{0}}\right) p_{\Gamma}(\gamma) \mathrm{d} \gamma,
\end{aligned}
$$

where $i \in\{0,1, \ldots, N\}, j \in\{1,2, \ldots, M\}, W$ is the system bandwidth, $P_{e}$ is the target bit-error-rate (BER), and recall that $P_{0}$ is the reference static transmit power. Note that we multiply the rate with $T_{f}$, which indicates that $\left.R(M i+j)\right|_{\mathbf{P}_{U}}$ is the number of bits that can be transmitted per frame. 


\section{Effective capacity analysis}

For a given power allocation policy $\mathbf{P}_{U}$, the sequence $\left\{R_{1}, R_{2}, \ldots\right\}$, where $R_{t} \in\left\{\left.R(1)\right|_{\mathbf{P}_{U}}, \ldots,\left.R((N+1) M)\right|_{\mathbf{P}_{U}}\right\}$, is a discrete-time stationary and ergodic service process. The effective capacity of the energy harvesting based service process with parameter $\theta$ is expressed as

$$
\alpha_{E C}(\theta)=-\lim _{K \rightarrow \infty} \frac{1}{\theta K T_{f}} \log \left(\mathbb{E}\left(e^{-\theta \sum_{t=1}^{K} R_{t}}\right)\right) .
$$

Theorem 1. Consider the system described in Sec. III. Denote $\boldsymbol{\Phi}\left(\theta, \mathbf{P}_{U}\right)=\operatorname{diag}\left\{e^{-\theta R(1) \mid \mathbf{P}_{U}}, \ldots, e^{-\theta R((N+1) M) \mid \mathbf{P}_{U}}\right\}$. If for a given power allocation policy $\mathbf{P}_{U}, \mathbf{P}_{S}=\mathbf{P}_{U}\left(\mathbf{P}_{A} \otimes \mathbf{P}_{C}\right)$ is irreducible and aperiodic, the effective capacity of the service process with energy harvesting is

$$
\alpha_{E C}(\theta)=-\frac{1}{\theta T_{f}} \log \left(\rho\left\{\boldsymbol{\Phi}\left(\theta, \mathbf{P}_{U}\right) \mathbf{P}_{U}\left(\mathbf{P}_{A} \otimes \mathbf{P}_{C}\right)\right\}\right)
$$

where $\rho\{\mathbf{A}\}$ is the spectral radius of matrix $\mathbf{A}$.

Proof. The proof follows the lines in [19, Example 3.3]. The details are presented in Appendix A.

It is obvious that the matrix $\mathbf{P}_{S}$ is aperiodic. However, it is not guaranteed irreducible for all policies $\mathbf{P}_{U}$. For instance, if $\mathbf{P}_{U}=\mathbf{I}_{(N+1) M}$ is an $(N+1) M \times(N+1) M$ unit matrix, i.e., the transmitter always uses 0 transmit power, the system will stays in states $(N, i), i=1, \ldots, M$. The following proposition provides the condition to guarantee the irreducibility.

Proposition 1. The Markov chain with the state transition matrix $\mathbf{P}_{S}$ is irreducible if and only if for all $i \geq 1, k \in$ $\{1, \ldots, M\}$, there exist two states $q<i \leq p$ such that $P_{U}^{(n)}(p, q, k)>0$ for some $n>0$, where $P_{U}^{(n)}(p, q, k)$ is the element of $\mathbf{P}_{U}^{n}$ with index $(p, q, k)$.

Proof. See Appendix B for details.

Based on Theorem 1, we can find the optimal policy by solving the effective capacity maximization problem as follows

$$
\begin{aligned}
\max _{\mathbf{P}_{U}} & -\frac{1}{\theta T_{f}} \log \left(\rho\left\{\boldsymbol{\Phi}\left(\theta, \mathbf{P}_{U}\right) \mathbf{P}_{U}\left(\mathbf{P}_{A} \otimes \mathbf{P}_{C}\right)\right\}\right) \\
\text { s.t. } & (14),(15), \text { and (16). }
\end{aligned}
$$

The problem (21) is an integer programming problem, which can be solved via exhausted search approach. Since the total number of possible power allocation policies is $((N+1) !)^{M}$, the complexity of finding the optimal solution grows at the speed much faster than exponent of the number of states $(N+1) M$. As a result, the exhausted search algorithm may not be applicable when the size of system state is large.

In this paper, we examine the effective capacity of the following power allocation policies:

1) Greedy policy: In the greedy power allocation policy, we simply use all the energy remained in the battery, i.e., $U_{t}=E_{t}$. Hence, the state transition matrix $\mathbf{P}_{U}$ can be expressed as

$$
\mathbf{P}_{U}=\left(\begin{array}{ll}
\mathbf{I}_{M} & \mathbf{0} \\
\vdots & \vdots \\
\mathbf{I}_{M} & \mathbf{0}
\end{array}\right) .
$$

The advantage of the greedy policy is that it minimizes the energy battery overflow (in this case, overflow only happens when the number of arrived energy units exceeds the battery size $N$ ). However, as the channel varies, this policy is very inefficient if a large amount of energy arrived while the channel is in deep fading.

2) Constant power policy: In this policy, the transmitter always transmit with a constant power as long as the required energy is available. Specifically, we set the constant number of energy units as $n_{C}$. If $E_{t} \geq n_{C}$, the energy $U_{t}=n_{C}$ is adopted. Otherwise, we set $U_{t}=0$. Hence, we have

$$
\mathbf{P}_{U}=\left(\begin{array}{ll}
\mathbf{I}_{n_{C} M} & \mathbf{0} \\
\mathbf{I}_{\left(N+1-n_{C}\right) M} & \mathbf{0}
\end{array}\right) .
$$

To balance battery energy depletion and overflow, we set the constant energy equal to the energy arrival $n_{C}=\lambda T_{f}$.

3) Constant-greedy policy: We also study the policy as [1, Eq. (4)] which is proved throughput optimal in AWGN channel with infinite battery capacity. The policy normally uses a constant transmit power. If the required power is not available, the remained energy is used up to support maximum rate. The policy is expressed as $U_{t}=\min \left\{n_{C}, E_{t}\right\}$. And the matrix expression of the policy is

$$
\mathbf{P}_{U}=\left(\begin{array}{ll}
\mathbf{I}_{M} & \mathbf{0} \\
\vdots & \vdots \\
\mathbf{I}_{M} & \mathbf{0} \\
\mathbf{I}_{\left(N+1-n_{C}\right) M} & \mathbf{0}
\end{array}\right) .
$$

As the remained energy will be used up if it is less than $n_{C}$, we name it by constant-greedy policy. Similar to the constant power policy, we set $n_{C}=\lambda T_{f}$.

4) Throughput optimal policy: In continuous fading channel, the throughput optimal (TO) policy is proved the WF policy [18]. In the discrete model without energy harvesting, the policy can be obtained by solving the following problem:

$$
\begin{array}{r}
\max _{U_{\mathrm{TO}}} \sum_{j=1}^{M} \pi_{j} R_{j}\left(u_{j}\right), \\
\text { s.t. } \sum_{j=1}^{M} \pi_{j} u_{j} \leq \bar{U}
\end{array}
$$

where $U_{\mathrm{TO}}=\left(u_{1}, \ldots, u_{M}\right), \pi_{i}$ is calculated as (11), and $R_{j}$ is calculated as (18) with $P(i, j)=u_{j} \Delta E / T_{f}$, where $u_{j}$ take all the values in $\{0,1, \ldots, N\}$ with average power constraint $\bar{U}$. When the energy harvesting is considered as in our model, the policy changes as: set $\bar{U}=\lambda T_{f}$ and solve the problem (25). If $E_{t} \geq u_{i}$ when $C_{t}=i$, we transmit with power $u_{i} \Delta E / T_{f}$. Otherwise, transmit power is set to zero.

We can also get the TO-greedy policy similarly as the constant-greedy one, i.e., $U_{t}=\min \left\{u_{i}, E_{t}\right\}$, if $C_{t}=i$.

\section{Numerical Results}

We study the performance of the considered power allocation policies by simulations. We set the frame length $T_{f}=2 \mathrm{~ms}$, the system bandwidth $W=10^{5} \mathrm{~Hz}$, the target BER $P_{e}=10^{-3}$, the Doppler spread $f_{d}=10 \mathrm{~Hz}$, the number of channel state $M=6$ and the Nakagami parameter $m=1$. The reference average SNR is obtained by using 


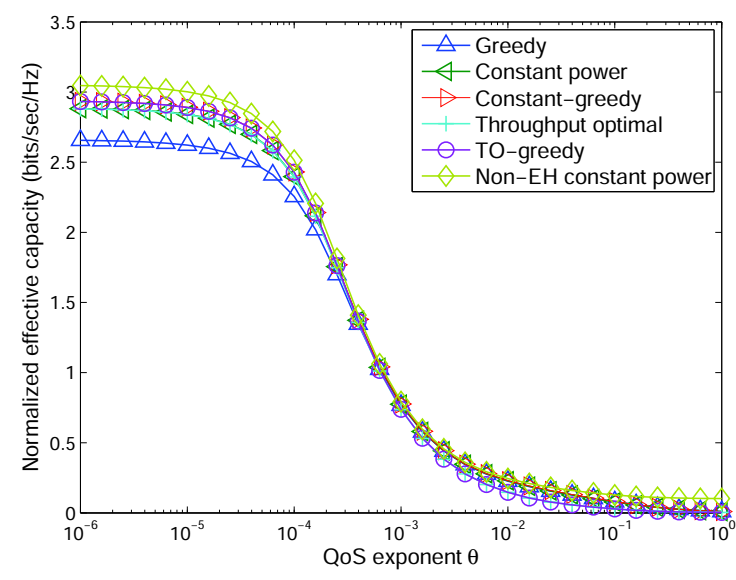

Fig. 2. The effective capacity comparison with $\bar{\gamma}=20 \mathrm{~dB}, \lambda T_{f}=2$.

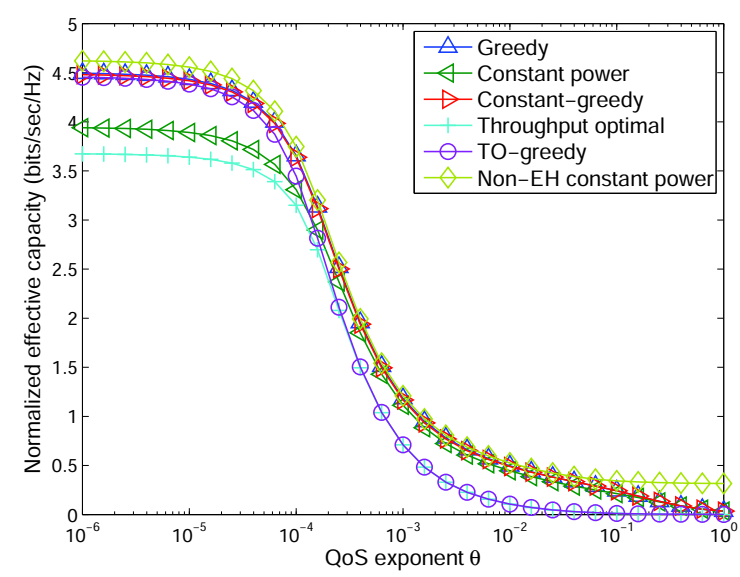

Fig. 3. The effective capacity comparison with $\bar{\gamma}=20 \mathrm{~dB}, \lambda T_{f}=7$.

power $P_{0}=5 \Delta E / T_{f}$. We fix the value of the battery capacity $N=10$ and change the energy arrival rate $\lambda$ to examine the influence of energy harvesting on effective capacity. We also change the reference average SNR $\bar{\gamma}$ to see how the channel fading interacts with the energy harvesting.

Figs. 2-4 show the effective capacity performance with different parameters. To illustrate the influence of random energy arrival, we compare with the constant power supply case and constant transmit power policy where $U_{t}=A_{t}=\lambda T_{f}$, i.e., no energy harvest (non-EH) constant power policy. Through all the simulation results, we find that energy harvesting based policies achieve lower effective capacity compared with the non-EH one.

The effective capacity performance of low energy arrival rate $\left(\lambda T_{f}=2\right)$ at high SNR regime $(20 \mathrm{~dB})$ is depicted in Fig. 2. The TO-greedy policy and the constant-greedy policy have the highest effective capacity at low QoS exponent regime $\left(\theta<5 \times 10^{-4}\right)$ which indicates a loose delay requirement. And the greedy policy performs the worst in this case. This

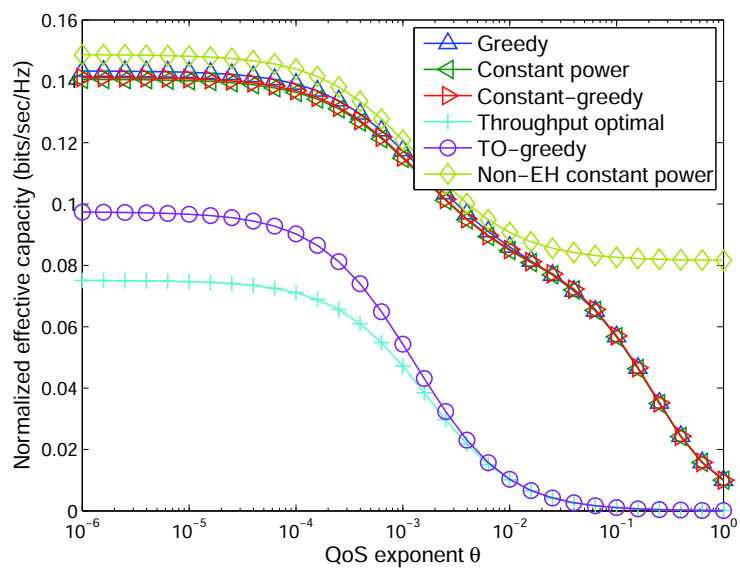

Fig. 4. The effective capacity comparison with $\bar{\gamma}=0 \mathrm{~dB}, \lambda T_{f}=2$.

is because the system tends to achieve maximum throughput when there is no constraints on delay. However, when $\theta>5 \times$ $10^{-4}$ which indicate a tight delay requirement, the throughput optimal policy and the TO-greedy policy perform the worst, even worse than the greedy policy. In this case, the data can not wait for good channel conditions, and should be transmitted as long as there is available power.

When the energy arrival rate is relatively high $\left(\lambda T_{f}=7\right.$ as shown in Fig. 3), we find that the throughput optimal policy performs the worst in all the conditions, and the constant power policy is the second worst when $\theta<1.5 \times 10^{-4}$. This is because the non-greedy policies waste a large amount of energy due to the battery overflow. Hence, greedy polices are preferred if the energy resource is rich. While at high QoS exponent regime, the performance is similar as in Fig. 2.

Finally, Fig. 4 shows the performance at low SNR regime $(0 \mathrm{~dB})$ and low arrival rate regime $\left(\lambda T_{f}=2\right)$. It is found that the greedy policy is the best one, while the throughput optimal policy and the TO-greedy policy performs much worse than the other three. The reason is that TO based policies allocate high power when the instantaneous SNR is high and low power when the SNR is low. However, the low SNR condition happens at high probability. As a result, the throughput optimal policy waits for high SNR condition and causes lots of battery overflows even though the energy arrival rate is low. Also, as data rate is sensitive to the power in low SNR regime, the constant-greedy policy performs slightly worse than the greedy policy due to its higher battery overflow probability.

\section{CONCLUSION}

In this work, we have established a discrete energy harvesting model and analyzed its QoS guarantee performance for given power allocation policies in terms of effective capacity. We derive the effective capacity formula and evaluate the performance of different policies according to the formula. Through numerical results, we find that the randomness of energy harvesting degrades the service rate compared with no 
energy harvesting case. Meanwhile, it is also shown that when the energy arrival rate is high or the channel condition is poor, the transmitter tends to use greedy based policies to reduce the energy overflow. And the constant-greedy policy achieves the highest effective capacity in high SNR regime.

\section{APPENDIX A \\ PROOF OF THEOREM 1}

Given the initial state $S_{1}=i$, the rate in this frame is determined as $R(i)$. Hence, we have

$$
\begin{aligned}
\psi(K, i) & =\mathbb{E}\left(e^{-\theta \sum_{k=1}^{K} R_{k}} \mid S_{1}=i\right) \\
& =e^{-\theta R(i)} \mathbb{E}\left(e^{-\theta \sum_{k=2}^{K} R_{k}} \mid S_{1}=i\right) \\
& =e^{-\theta R(i)} \sum_{j=1}^{(N+1) M} P_{S}(i, j) \mathbb{E}\left(e^{-\theta \sum_{k=2}^{K} R_{k}} \mid S_{2}=j\right) \\
& =e^{-\theta R(i)} \sum_{j=1}^{(N+1) M} P_{S}(i, j) \psi(K-1, j),
\end{aligned}
$$

where (27) follows the law of total probability and the fact that the rate $R_{k}$ only depends on the state $S_{k}$, and in (28), we just replace the time index $k$ by $k-1$. As a result, we have

$$
\Psi(K)=\boldsymbol{\Phi}(\theta) \mathbf{P}_{S} \Psi(K-1),
$$

where $\Psi(K)=(\psi(K, 1), \psi(K, 2), \ldots, \psi(K,(N+1) M))^{T}$ and $\boldsymbol{\Phi}(\theta)=\boldsymbol{\Phi}\left(\theta, \mathbf{P}_{U}\right)$ for simplicity. Denote $\tilde{\pi}_{i}=\operatorname{Pr}\left(S_{1}=i\right)$ as the probability that the state in frame 1 being $i$, and let $\Pi=\left(\tilde{\pi}_{1}, \tilde{\pi}_{2}, \ldots, \tilde{\pi}_{(N+1) M}\right)$, we can get

$$
\mathbb{E}\left(e^{-\theta \sum_{k=1}^{K} R_{k}}\right)=\Pi \Psi(K)=\Pi\left(\boldsymbol{\Phi}(\theta) \mathbf{P}_{S}\right)^{K-1} \mathbf{\Phi}(\theta) \mathbf{1},
$$

where $\mathbf{1}$ is an $(N+1) M \times 1$ vector with all elements equal 1. As the state transition matrix $\mathbf{P}_{S}$ is irreducible and aperiodic with non-negative elements, and $\boldsymbol{\Phi}(\theta)$ is a diagonal matrix with positive diagonal elements, we get that the matrix $\boldsymbol{\Phi}(\theta) \mathbf{P}_{S}$ is primitive, i.e., it is non-negative and for some $k \geq 1$, the elements of $\left(\boldsymbol{\Phi}(\theta) \mathbf{P}_{S}\right)^{k}$ are all positive. According to the Perron-Frobenius theorem [20, Theorem 8.5.1], i.e., there exists a limit $\left(\boldsymbol{\Phi}(\theta) \mathbf{P}_{S} / \rho\left\{\boldsymbol{\Phi}(\theta) \mathbf{P}_{S}\right\}\right)^{k}$ for $k \rightarrow \infty$ with positive elements, we have

$$
\begin{gathered}
\alpha_{E C}=-\frac{1}{\theta T_{f}} \lim _{K \rightarrow \infty} \log \left(\rho\left\{\boldsymbol{\Phi}(\theta) \mathbf{P}_{S}\right\}^{\frac{K-1}{K}} .\right. \\
\left.\left(\Pi\left(\frac{\boldsymbol{\Phi}(\theta) \mathbf{P}_{S}}{\rho\left\{\boldsymbol{\Phi}(\theta) \mathbf{P}_{S}\right\}}\right)^{K-1} \boldsymbol{\Phi}(\theta) \mathbf{1}\right)^{\frac{1}{K}}\right) .
\end{gathered}
$$

It turns out $\alpha_{E C}$ is expressed as (20).

\section{APPENDIX B \\ PROOF OF PROPOSITION 1}

As the channel state transition is irreducible and independent of the battery state transition, we only need to consider the latter. Hence, we ignore the index $k$ for simplicity.

Sufficiency. Firstly, any state $i$ is accessible from any state $j \leq i$ as $P_{A}(j, i)>0$. On the other hand, with the given condition, we can find states $q<i \leq p$ so that $P_{U}^{(n)}(p, q)>0$.
As $P_{A}(i, p)>0$, state $q<i$ is accessible from $i$. Repeating the same process, we can get that state 0 is accessible from $i$. Hence, $j$ is accessible from $i$ as $P_{A}(0, j)>0$, which proves the irreducibility.

Necessity. If the condition does not hold, i.e., $\exists i>0, \forall j \geq$ $i, P_{U}^{(n)}(j, p)=0, \forall p<i, n>0$, combining with the condition that $P_{A}^{(n)}(j, p)=0$, the states $p<i$ is not accessible from states $j \geq i$. As a result, the Markov chain is reducible.

\section{REFERENCES}

[1] V. Sharma, U. Mukherji, V. Joseph, and S. Gupta, "Optimal energy management policies for energy harvesting sensor nodes," IEEE Transactions on Wireless Communications, vol. 9, no. 4, pp. 1326-1336, 2010.

[2] O. Ozel and S. Ulukus, "Achieving awgn capacity under stochastic energy harvesting," IEEE Transactions on Information Theory, vol. 58, no. 10, pp. 6471-6483, 2012.

[3] V. Sharma, R. Rajesh et al., "Awgn channel capacity of energy harvesting transmitters with a finite energy buffer," arXiv preprint arXiv:1307.4505, 2013.

[4] O. Ozel, K. Tutuncuoglu, J. Yang, S. Ulukus, and A. Yener, "Transmission with energy harvesting nodes in fading wireless channels: Optimal policies," IEEE Journal on Selected Areas in Communications, vol. 29, no. 8 , pp. 1732-1743, 2011.

[5] C. Hu, J. Gong, X. Wang, S. Zhou, and Z. Niu, "Spatial-temporal water-filling power allocation in mimo systems with harvested energy," in IEEE/CIC International Conference on Communications in China (ICCC). IEEE, 2013, pp. 588-593.

[6] J. Gong, S. Zhou, and Z. Niu, "Optimal power allocation for energy harvesting and power grid coexisting wireless communication systems," IEEE Transactions on Communications, vol. 61, no. 7, pp. 3040-3049, 2013.

[7] D. Wu and R. Negi, "Effective capacity: a wireless link model for support of quality of service," IEEE Transactions on Wireless Communications, vol. 2, no. 4, pp. 630-643, 2003.

[8] J. Tang and X. Zhang, "Quality-of-service driven power and rate adaptation over wireless links," IEEE Transactions on Wireless Communications, vol. 6, no. 8, pp. 3058-3068, 2007.

[9] D. Wu and R. Negi, "Power control and scheduling for guaranteeing quality of service in cellular networks," Wireless Communications and Mobile Computing, vol. 8, no. 1, pp. 75-92, 2008.

[10] X. Zhang and J. Tang, "Power-delay tradeoff over wireless networks," IEEE Transactions on Communications, vol. 61, no. 9, pp. 3673-3684, 2013.

[11] J. Tang and X. Zhang, "Cross-layer modeling for quality of service guarantees over wireless links," IEEE Transactions on Wireless Communications, vol. 6, no. 12, pp. 4504-4512, 2007.

[12] Q. Wang, D. Wu, and P. Fan, "Effective capacity of a correlated rayleigh fading channel," Wireless Communications and Mobile Computing, vol. 11, no. 11, pp. 1485-1494, 2011.

[13] A. Goldsmith, Wireless communications. Cambridge University Press, 2005.

[14] H. S. Wang and N. Moayeri, "Finite-state markov channel-a useful model for radio communication channels," IEEE Transactions on Vehicular Technology, vol. 44, no. 1, pp. 163-171, 1995.

[15] Q. Zhang and S. A. Kassam, "Finite-state markov model for rayleigh fading channels," IEEE Transactions on Communications, vol. 47, no. 11, pp. 1688-1692, 1999.

[16] M. D. Yacoub, J. V. Bautista, and L. Guerra de Rezende Guedes, "On higher order statistics of the nakagami-m distribution," IEEE Transactions on Vehicular Technology, vol. 48, no. 3, pp. 790-794, 1999.

[17] R. A. Horn and C. R. Johnson, Topics in matrix analysis. Cambridge University Press, 1991.

[18] A. J. Goldsmith and S.-G. Chua, "Variable-rate variable-power mqam for fading channels," IEEE Transactions on Communications, vol. 45, no. 10 , pp. 1218-1230, 1997.

[19] C.-S. Chang, "Stability, queue length, and delay of deterministic and stochastic queueing networks," IEEE Transactions on Automatic Control, vol. 39, no. 5, pp. 913-931, 1994.

[20] R. Horn and C. Johnson, Matrix Analysis. Cambridge University Press, 1985. 